\title{
Generalist predator dynamics under Kolmogorov versus non-Kolmogorov models
}

\author{
Yifang $\mathrm{Xu}^{1}$, Andrew L. Krause ${ }^{1}$, and Robert A. Van Gorder ${ }^{2, *}$ \\ ${ }^{1}$ Mathematical Institute, University of Oxford, Andrew Wiles Building, Radcliffe Observatory Quarter, Woodstock Road, Oxford, OX2 6GG, UK \\ ${ }^{2}$ Department of Mathematics and Statistics, University of Otago, P.O. Box 56, Dunedin 9054, New Zealand \\ *rvangorder@maths.otago.ac.nz
}

\begin{abstract}
Ecosystems often contain multiple species across two or more trophic levels, with a variety of interactions possible. In this paper we study two classes of models for generalist predators that utilize more than one food source. These models fall into two categories: predator - two prey and predator - prey - subsidy models. For the former, we consider a generalist predator which utilizes two distinct prey species, modelled via a Kolmogorov system of equations with Type II response functions. For the latter, we consider a generalist predator which exploits both a prey population and an allochthonous resource which is provided as a subsidy to the system exogenously, again with Type II response functions. This latter class of model is no longer Kolmogorov in form, due to an exogenous forcing term modelling the input of the allochthonous resource into the system. We non-dimensionalize both models, so that their respective parameter spaces may be more easily compared, and study the dynamics possible from each type of model, which will then indicate - for specific parameter regimes - which generalist predator's preferences are more favorable to survival, including the prevalence of coexistence states. We also consider the various non-equilibrium dynamics emergent from such models, and show that the non-Kolmogorov predator - prey - subsidy model often admits more regular dynamics (including steady states and one type of limit cycle), whereas the predator - two prey Kolmogorov model can feature multiple types of limit cycles, as well as multistability resulting in strong sensitivity to initial conditions (with stable limit cycles and steady states both coexisting for the same model parameters). Our results highlight several interesting differences and similarities between Kolmogorov and non-Kolmogorov models for generalist predators.
\end{abstract}

Keywords: predator-prey interactions, generalist predator, Kolmogorov predator-prey model, non-Kolmogorov predator-prey model

\section{Introduction}

Generalist predators make use of more than one food source: either multiple prey species, or a combination of prey species and allochthonous resources. While there is a rich theoretical literature on generalist predators (Erbach et al., 2013; Morozov and Petrovskii, 2013; Parshad et al., 2016; Rutz and Bijlsma, 2006; Sanders et al., 2011; Smout et al., 2010; Sunde et al., 2014; Terraube and Arroyo, 2011), there are also a variety of real applications for such models (Clare et al., 2009; Díaz-Ruiz et al., 2013; Weber and Lundgren, 2011; Willson and Hopkins, 2011), with particular relevance to the study of sustainability or control of food webs (Kar and Ghosh, 2012; Maron et al., 2010; Nomikou et al., 2010; Snyder and Wise, 1999; Symondson et al., 2002; van Maanen et al., 2010; Welch et al., 2012). Here we will study the impact that different kinds of predation have on the dynamics of systems with generalist predators.

The competitive exclusion principle (Gause's law) states that two identical species cannot coexist unless they are different in at least one aspect (Vance, 1978), yet introducing a predator to a system of two competing prey species allows them to coexist in some settings (Vance, 1978). Many one predator-two interacting prey models, where the two prey either compete or help each other, have been studied (Abrams and Matsuda,
1993; Leeuwen et al., 2007; Liu and Wang, 2013; Mariani et al., 2013; Zhang et al., 2015). Gilpin (1979) studied dynamics from a three-species ecosystem, and later work considered logistic growth of the prey populations in such models to account for resource limitations (El-Gohary and Al-Ruzaiza, 2007; Gakkhar et al., 2003; Hutson and Vickers, 1983; Klebanoff and Hastings, 1994; Kumar et al., 2002; Takeuchi and Adachi, 1983). In such models, the populations can approach a steady state, fall into a limit cycle, or exhibit chaotic dynamics. A similar model is the prey-predator-super predator model, where a predator exploits a prey, and a super predator exploits the predator, yet has no relationship with the prey (Hastings and Powell, 1991). The importance of understanding aperiodic or chaotic dynamics emergent from ecological models was discussed in Schaffer and Kot (1986), where it was suggested that rather than being random and disorganized, the structure of chaotic trajectories can contain important information about the dynamics of ecological systems relevant to explaining real ecosystem dynamics.

While generalist predator systems in which a predator can utilize two or more prey species are commonly found in nature, so are situations where a predator utilizes an allochthonous resource in addition to prey (Giroux et al., 2012; Legagneux et al., 2012; Marczak et al., 2007; Rose and Polis, 1998). Population dynamics have been shown to be altered when predators utilize 


\begin{tabular}{|c|c|c|c|}
\hline Behavior & $\left(x^{*}, y^{*}\right)$ & Feasibility & Stability \\
\hline Extinction SS & $(0,0)$ & Always & Never \\
\hline Predator Free SS & $(K, 0)$ & Always & $D>\frac{K}{1+P K}$ \\
\hline Positive SS & $\left(\frac{D}{1-D P}, \frac{1}{1-D P}\left(1-\frac{D}{(1-D P) K}\right)\right)$ & $1-D P>0, K>\frac{D}{(1-D P)}$ & $K<\frac{1+D P}{P(1-P D)}$ \\
\hline Limit Cycle & - & $1-D P>0, K>\frac{1+D P}{P(1-P D)}$ & When Feasible \\
\hline
\end{tabular}

Table 1: List of the steady states (SS), along with feasibility and stability conditions, for the non-dimensional system (2a)-(2b). We also provide conditions for the existence of limit cycles.

subsidies of natural resources (Darimont et al., 2008; Halaj and Wise, 2002; Henden et al., 2010). These systems have been modelled using exogeneous forcing representing additional resources flowing into the system (Nevai and Van Gorder, 2012), and so do not obey the typical form of Kolmogorov systems (for which the rate of change of each population is purely densitydependent). In addition to exogenous resources, there have also been studies which couple predator-prey dynamics to temperature (Wollkind et al., 1988; Nomdedeu et al., 2012). Hence, there are a range of external effects which might be modelled through a non-Kolmogorov framework.

From the aforementioned applications, it is clear that natural ecosystems often contain more than two species or two levels, and we shall be interested in studying two classes of models for generalist predators which utilize more than one food source. In Section 2, we review the dynamics of the RosenzweigMacArthur model, as we build on this model. In Section 3 we consider a generalist predator which utilizes two distinct prey species, modelled via a Kolmogorov system (Kolmogorov, 1936; Sigmund, 2007) of equations with Holling Type II functional response (Holling, 1959). In Section 4 we consider a generalist predator which exploits both a prey population and an allochthonous resource which is provided as a subsidy to the system, again with Type II response functions. This latter class of model is no longer Kolmogorov in form, due to an exogenous forcing term modelling the input of the allochthonous resource into the system. While each type of model has been considered in the literature, it is useful to consider how the dynamics of each type of model will differ, and we non-dimensionalize both models in a similar manner so that their respective parameter spaces are comparable. We classify the dynamics emergent from each model, and contrast the steady states and nonequilibrium dynamics (such as limit cycles and multistability) inherent from each family of models. We discuss our findings in Section 5.

\section{Review of Rosenzweig-MacArthur dynamics}

A commonly employed model for predator-prey interactions takes the form

$$
\begin{gathered}
\frac{\mathrm{d} x}{\mathrm{~d} t}=r x\left(1-\frac{x}{k}\right)-\frac{\theta x y}{x+h}, \\
\frac{\mathrm{d} y}{\mathrm{~d} t}=\frac{\epsilon \theta x y}{x+h}-\delta y,
\end{gathered}
$$

where $x$ represents the prey population and $y$ represents the predator population. The prey grows at a constant rate $r$, the predator consumes the prey at the rate $\theta$, the predator benefits from the prey at the rate $\epsilon$, and the predator population dies at the rate $\delta$. Here the intrinsic growth rate of the prey is modelled by a logistic term, and hence the prey population can never exceed the carrying capacity, $k$. The rate at which the predator consumes the prey is modelled with a Holling Type II functional response $\theta x /(x+h)$ (Holling, 1959, 1966; Turchin, 2003). This ensures that the prey consumption rate saturates as the prey population grows large. Rosenzweig and MacArthur (1963) represented the predator-prey interactions graphically and provided stability conditions for the two species interaction, and the model (1) is often referred to as the Rosenzweig-MacArthur model. A more analytical approach for global stability of the steady states was later provided by Hsu (1978). When $k$ is sufficiently small, the model indicates a stable predator-free equilibrium. For a certain range of the carrying capacity, $k$, this model admits a stable steady state solution, which attracts all positive initial populations. As $k$ increases beyond this range, a supercritical Hopf-bifurcation will occur, which leads to limit cycle dynamics (Hofbauer and Sigmund, 1998; Kot, 2001; Nevai and Van Gorder, 2012). As $k$ increases further, the maximum of the prey population increases, but the minimum of the prey population may decrease to vanishingly small values which cannot persist in nature (Kot, 2001). Rosenzweig referred to this nonintuitive phenomena, in which the prey is enriched yet which the populations die off, as the paradox of enrichment (Rosenzweig, 1971).

We non-dimensionalize all models considered in this paper, so as to better compare solutions between the models. To nondimensionalize (1), we scale $t=\bar{t} \hat{t}, x=\bar{x} \hat{x}, y=\bar{y} \hat{y}$. Adopting the scalings $\bar{t}=\frac{1}{r}, \bar{y}=\frac{h}{\theta \bar{t}}=\frac{h r}{\theta}, \bar{x}=\frac{h}{\epsilon \theta t}=\frac{h r}{\epsilon \theta}$, we reduce the parameter space to the positive dimensionless groups $K=\frac{\epsilon \theta k}{h r}$ (effective carrying capacity), $D=\frac{\delta}{r}$ (effective death rate), $P=$ $\frac{r}{\epsilon \theta}$ (rate of prey renewal to exploitation). With these scalings, we have that $D \ll 1, K, P=O(1)$ for ecologically sensible parameter ranges. Dropping hats on the variables, we obtain the non-dimensional form of (1), which reads

$$
\begin{gathered}
\frac{\mathrm{d} x}{\mathrm{~d} t}=x\left(1-\frac{x}{K}\right)-\frac{x y}{1+P x}, \\
\frac{\mathrm{d} y}{\mathrm{~d} t}=\frac{x y}{1+P x}-D y .
\end{gathered}
$$

The feasibility and stability of the three steady states $\left(x^{*}, y^{*}\right)$ of (2) are listed in Table 1. One may show that solutions of (2) remain positive and bounded. When all steady states lose stability, the trajectories enter into a positively invariant set which 
does not border any steady states nor contain any stable steady states, so by the Poincaré - Bendixson theorem, the only nonequilibrium behaviors possible are limit cycles.

Taking the limit $P \ll 1$, (2) reduces to the simplified model

$$
\begin{gathered}
\frac{\mathrm{d} x}{\mathrm{~d} t}=x\left(1-\frac{x}{K}\right)-x y, \\
\frac{\mathrm{d} y}{\mathrm{~d} t}=x y-D y,
\end{gathered}
$$

where the interspecies interaction terms are now Type I functional responses. Biologically, the $P \ll 1$ limit ( i.e., $r \ll \epsilon \theta$ ) means that the rate at which the predator exploits the prey is much larger than the intrinsic growth or renewal rate of the prey. This model is essentially the Lotka-Volterra model with the linear prey growth term modified to a logistic growth term. We list the stability and feasibility of the steady states to (3) in Table 2. We find that the dynamics of the simplified model (3) are also bounded. While this model is less biologically relevant, as the exploitation rate is more commonly less than the renewal rate for populations which persist, it is a far easier and more pedagogical model by which to understand steady state dynamics.

\begin{tabular}{|c|c|c|c|}
\hline Behavior & $\left(x^{*}, y^{*}\right)$ & Feasibility & Stability \\
\hline Extinction & $(0,0)$ & Always & Never \\
\hline Predator Free & $(K, 0)$ & Always & $K<D$ \\
\hline Positive & $\left(D, 1-\frac{D}{K}\right)$ & $K>D$ & When Feasible \\
\hline
\end{tabular}

Table 2: Steady states, feasibility and stability conditions for the system (3).

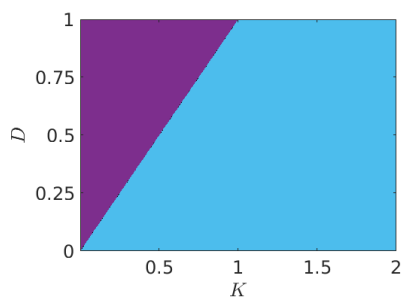

(a) $P=0$

Predator-free SS

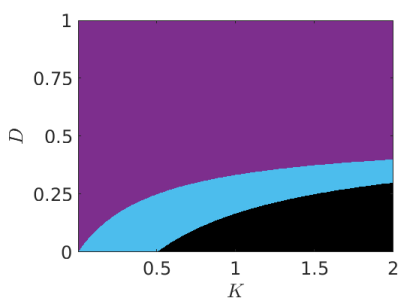

(b) $P=2$
Figure 1: Comparison of dynamics between the (a) simplified model (3) and (b) Rosenzweig-MacArthur model (2) with $P=2$. The plots are generated using the information from Table 1 and 2. Simulations from the black region always give limit cycles.

Both the Rosenzweig-MacArthur model (2) and the simplified model (3) have three steady states, although the extinction state is never stable. Figure 1 displays the change of stability regions when comparing the dynamics between the models (2) and (3). When $D=K$, a transcritical bifurcation occurs for model (3), and likewise for model (2) when $D=K /(1+P K)$. Any positive value of $P$ in (2) results in at least some parameters permitting non-equilibrium dynamics resulting in limit cycles (along the border of this region, there is a Hopf bifurcation curve), while non-equilibrium dynamics do not occur for (3). The Rosenzweig-MacArthur model (2) has limit cycle solutions when $K$ is sufficiently large (although in many ecological systems, the carrying capacity is not always very large, as discussed in Hobbs and Swift (1985)). Hence, the $P=0$ limit modelling a Type 1 functional response is degenerate from the $P=O(1)$ regime of the Type II functional response, representing a distinct distinguished limit within which for all parameter values there will always be a stable steady state (either predator free or positive).

\section{Kolmogorov model for generalist predators utilizing two prey}

An example of a predator-two prey system consists of the mountain lion predating on both mule deer and porcupine in North America's Great Basin Desert (Sweitzer et al., 1997). Sweitzer et al. (1997) suggested that changes in plant communities resulted in the irruption (explosive growth) of mule deer and corresponding expansion of mountain lions. The increased population of mountain lion then caused the near extinction of porcupines, which is a native species. A further case study on this ecosystem was carried out by Grasman et al. (2001). Similar dynamics occur in predator - prey dynamics involving wolves which exploit increased moose populations due to clearcutting (Fisher and Wilkinson, 2005; Hins et al., 2009; Houle et al., 2010), with this resulting in additional stress on the caribou population which is also exploited by the wolves. As another example, Hamilton (1935) demonstrated that the red fox predates independent prey, including squirrels and rabbits. Such models also arise in the biological control of thrips and whiteflies by a shared predator (Messelink et al., 2008).

The predator-two prey model we consider takes the form

$$
\begin{gathered}
\frac{\mathrm{d} x}{\mathrm{~d} t}=r_{1} x\left(1-\frac{x}{k_{1}}\right)-\frac{\theta x y}{x+w+h}, \\
\frac{\mathrm{d} w}{\mathrm{~d} t}=r_{2} w\left(1-\frac{w}{k_{2}}\right)-\frac{\psi w y}{x+w+h}, \\
\frac{\mathrm{d} y}{\mathrm{~d} t}=\frac{\epsilon \theta x y}{x+w+h}+\frac{\eta \psi w y}{x+w+h}-\delta y,
\end{gathered}
$$

where $x, w$ denote the population of prey 1 and prey 2, respectively, while $y$ denotes the generalist predator population. Here $r_{1}, r_{2}$ are the intrinsic growth rates of prey 1 and prey $2, k_{1}, k_{2}$ are the carrying capacities of prey 1 and prey $2, \theta, \psi$ are the maximum rates at which the predators exploit prey 1 and prey 2 , and $\epsilon, \eta$ are the conversion rates of the energy that the predator obtains from prey 1 and prey 2 , respectively. The handling time $h$ is assumed to be uniform over all food sources. Other parameters are the same as in the Rosenzweig-MacArthur model (1). Note that (4) is an example of a Kolmogorov generalist predator model with independent prey. Models of this type have been studied previously (Fussell et al., 2019; Genkai-Kato and Yamamura, 2000; Yamauchi and Yamamura, 2005; Abrams, 2006; Mougi and Nishimura, 2008; Mougi, 2010; Toyokawa, 2017). We note that while the assumption of independent prey is restrictive, it allows us to compare the qualitative impact of different predation strategies in a simple setting with the model in Section 4.

We non-dimensionalize the system variables with $t=\bar{t} \hat{t}$, $x=\bar{x} \hat{x}, w=\bar{w} \hat{w}$ and $y=\bar{y} \hat{y}$, and choose the scalings $\bar{t}=\frac{1}{r_{1}}$, 


\begin{tabular}{|c|c|c|}
\hline Behavior & $\left(x^{*}, w^{*}, y^{*}\right)$ & Feasibility \\
\hline Extinction & $(0,0,0)$ & Always \\
\hline Prey 1 Only & $\left(K_{1}, 0,0\right)$ & Always \\
\hline Prey 2 Only & $\left(0, K_{2}, 0\right)$ & Always \\
\hline Predator-Free & $\left(K_{1}, K_{2}, 0\right)$ & $D<K_{1}+K_{2}$ \\
\hline Prey 1-Free & $\left(0, \frac{D}{1-D Q}, \frac{1}{\Phi}\left(1+\frac{Q D}{1-Q D}\right)\left(R-\frac{R D}{K_{2}(1-D Q)}\right)\right)$ & $1-D Q>0, K_{2}>\frac{D}{1-D Q}$ \\
\hline Prey 2-Free & $\left(\frac{D}{1-D P}, 0, \frac{1}{1-D P}-\frac{D}{K_{1}(1-D P)^{2}}\right)$ & $1-D P>0, K_{1}>\frac{D}{1-D P}$ \\
\hline Positive & $\left(x^{*}, w^{*}, y^{*}\right)$ & $x^{*}>0, w^{*}>0, y^{*}>0$ \\
\hline
\end{tabular}

Table 3: Steady states and feasibility conditions for the predator-two prey model (5).

$\bar{y}=\frac{r_{1} h}{\theta}, \bar{x}=\frac{r_{1} h}{\epsilon \theta}, \bar{w}=\frac{r_{1} h}{\eta \psi}$. We define the dimensionless groups $K_{1}=\frac{\epsilon \theta k_{1}}{h r_{1}}, K_{2}=\frac{\eta \psi k_{2}}{h r_{1}}, \Phi=\frac{\psi}{\theta}, D=\frac{\delta}{r_{1}}, R=\frac{r_{2}}{r_{1}}, P=\frac{r_{1}}{\epsilon \theta}, Q=$ $\frac{r_{1}}{\eta \psi}$. Note that $K_{1}, K_{2}=O(1)$ are effective carrying capacities, $D \ll 1$ is an effective death rate, $R=O(1)$ is the ratio of growth or renewal rates from each prey species, while $P, Q=O(1)$ are respective rates of renewal to exploitation for each prey. The parameter $\Phi$ measures the preference of the generalist predator for each prey species, with $\Phi>1$ indicating a preference for prey 2 and $\Phi<1$ indicating a preference for prey 1 . When $\Phi=1$, both prey are preferred equally. Upon dropping the hats, the dimensionless form of the system (4) reads

$$
\begin{gathered}
\frac{\mathrm{d} x}{\mathrm{~d} t}=x\left(1-\frac{x}{K_{1}}-\frac{y}{1+P x+Q w}\right), \\
\frac{\mathrm{d} w}{\mathrm{~d} t}=w\left(R-\frac{R w}{K_{2}}-\frac{\Phi y}{1+P x+Q w}\right), \\
\frac{\mathrm{d} y}{\mathrm{~d} t}=y\left(\frac{x+w}{1+P x+Q w}-D\right) .
\end{gathered}
$$

The dynamics of (5) may be shown to be bounded. In Table 3, we summarize the steady states and their feasibility conditions.

Determining stability of equilibria for the predator-two prey model is not analytically tractable, so we compute the Jacobian at each steady state and then determine the stability numerically, via numerical evaluation of the eigenvalues of the Jacobian. Since the extinction, prey 1 only, and prey 2 only steady states are never stable, only the predator-free, prey 1-free, prey 2-free, and positive steady states will appear in the bifurcation diagrams. While standard bifurcation methods and software exists to perform such analysis (e.g. (Doedel, 1981; Dhooge et al., 2003; Seydel, 2009)), due to the complexity of visualizing basins of attraction of different attracting sets, we will use direct numerical simulations for ease of analysis when dealing with non-equilibrium states, such as limit cycles. In particular, we take the initial condition to be the positive steady state plus a normally distributed perturbation with standard deviation $10^{-3}$ (taking absolute values in the case that the positive steady state is not feasible), and evolve this solution for a long time period $(t \leq 3000)$ by which point all trajectories have reached their long-time behaviours. In this way, we gain an understanding of what happens to a coexistence equilibrium if system parameters change quasi-statically due to external fluctuations, such as environmental change.

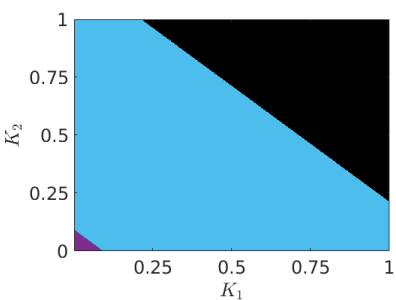

(a) $P=1, Q=1$

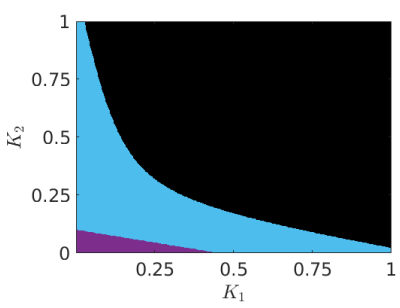

(c) $P=8, Q=1$

Predator-free SS

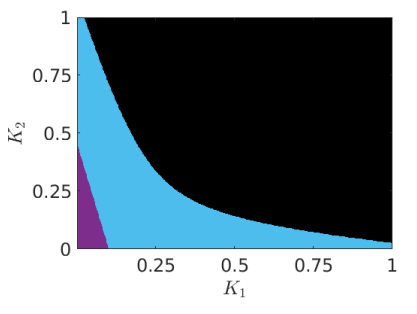

(b) $P=1, Q=8$

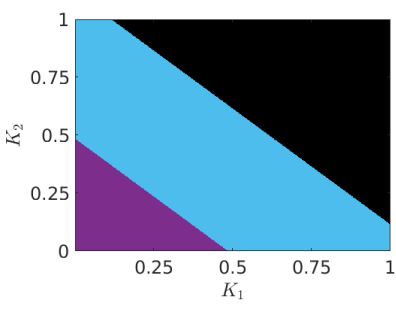

(d) $P=8, Q=8$

Non-equilibrium dynamics
Figure 2: Bifurcation diagrams over $K_{1}, K_{2}$ for the steady states and nonequilibrium dynamics of the predator-two prey model (5) given various $P, Q$. Parameters $D=0.1, R=1, \Phi=1$ are fixed.

Figure 2 shows the change of stability regions for each of the emergent behaviors (predator-free and positive steady states in addition to non-equilibrium dynamics consisting of limit cycles) when we change the values of the ratios of prey renewal to exploitation, $P$ and $Q$. As the prey are likely be renewed at rates similar to or greater than exploitation, we take $P, Q=O(1)$ or larger. However, it is possible to have values smaller than one, in either an unhealthy system, or with one of $P$ or $Q$ large enough to counter the other being small. When the values of $P$ and $Q$ are equal and the preference for each prey is equal to one, the corresponding regions for the predator-free steady state, positive steady state, and non-equilibrium dynamics are symmetric about the line $K_{1}=K_{2}$ in parameter space. The dynamics are akin to what is observed for the RosenzweigMacArthur model (2). When $P$ and $Q$ are not equal, then the dynamics may be skewed toward one or the other prey species, but the qualitative behaviors observed are consistent.

In order to better understand how the preference parameter, $\Phi$, influences the dynamics emergent from (5), we consider bifurcation diagrams for different values of $\Phi$ in Figure 3, considering the case of $\Phi<1$ (so that prey 1 is preferred over 


\begin{tabular}{|c|c|c|c|}
\hline Behavior & $\left(x^{*}, w^{*}, y^{*}\right)$ & Feasibility & Stability \\
\hline Extinction & $(0,0,0)$ & Always & Never \\
\hline Prey 1 Only & $\left(K_{1}, 0,0\right)$ & Always & Never \\
\hline Prey 2 Only & $\left(0, K_{2}, 0\right)$ & Always & Never \\
\hline Predator Free & $\left(K_{1}, K_{2}, 0\right)$ & Always & $D>K_{1}+K_{2}$ \\
\hline Prey 1 Free & $\left(0, D, \frac{R K_{2}-R D}{K_{2} \Phi}\right)$ & $D<K_{2}$ & $\Phi<R\left(1-\frac{D}{K_{2}}\right)$ \\
\hline Prey 2 Free & $\left(D, 0,1-\frac{D}{K_{1}}\right)$ & $D<K_{1}$ & $\Phi>R\left(1-\frac{D}{K_{1}}\right)^{-1}$ \\
\hline Positive & $\left(x^{*}, w^{*}, y^{*}\right)$ & $R\left(1-\frac{D}{K_{2}}\right)<\Phi<R\left(1-\frac{D}{K_{1}}\right)^{-1}, D<K_{1}+K_{2}$ & When Feasible \\
\hline
\end{tabular}

Table 4: Steady states, feasibility and stability conditions for the simplified predator-two prey model (6). Note that the feasibility conditions for the positive steady state are the complement of the stability conditions for the other steady states. The positive steady state is stable when feasible, hence there will be exactly one stable steady state for all parameter values.

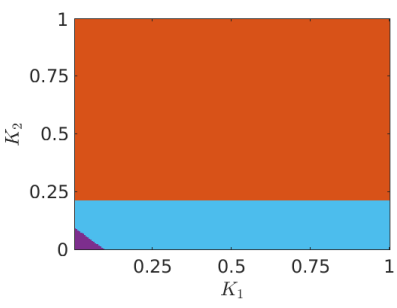

(a) $P, Q=1, \Phi=0.5$

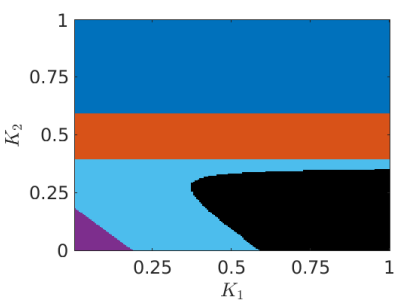

(c) $P, Q=5, \Phi=0.5$

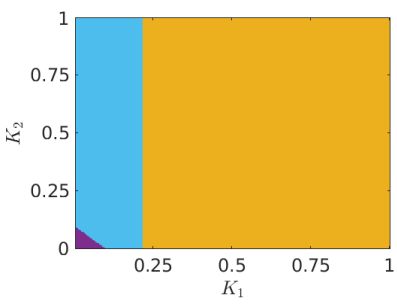

(b) $P, Q=1, \Phi=2$

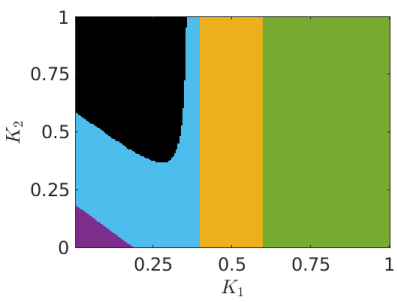

(d) $P, Q=5, \Phi=2$

Prey 1-free SS Prey 2-free SS

Predator-free SS Positive SS
Prey 1 - Predator non-equilibrium dynamics

Prey 2 - Predator non-equilibrium dynamics

Prey 1 - Prey 2 - Predator non-equilibrium dynamics

Figure 3: Bifurcation diagrams over $K_{1}, K_{2}$ for the steady states and nonequilibrium dynamics of the predator-two prey model (5) given various $P, Q$, and $\Phi$. We fix $D=0.1, R=1$.

prey 2) and $\Phi>1$ (so that prey 2 is preferred over prey 1 ). Here the coupling between the preference and the renewal to exploitation rates will matter in determining which dynamics are possible. In addition to the various dynamics observed in Figure 2, we now observe the possibility of asymmetric states where one of the two prey species is extinct. These may either consist of a steady state for which one of the prey species is extinct, or limit cycles between the predator and one prey, with the other prey having gone extinct. As such, the non-equilibrium dynamics no longer necessarily involve all three species, but may involve only pairs of species, with the other prey population equal to zero. We therefore clarify which of the behaviors we observe in each region, by plotting three distinct regions of non-equilibrium dynamics.

We find that certain parameter combinations permit multistability in the dynamics of (5). In Figure 4, we provide an

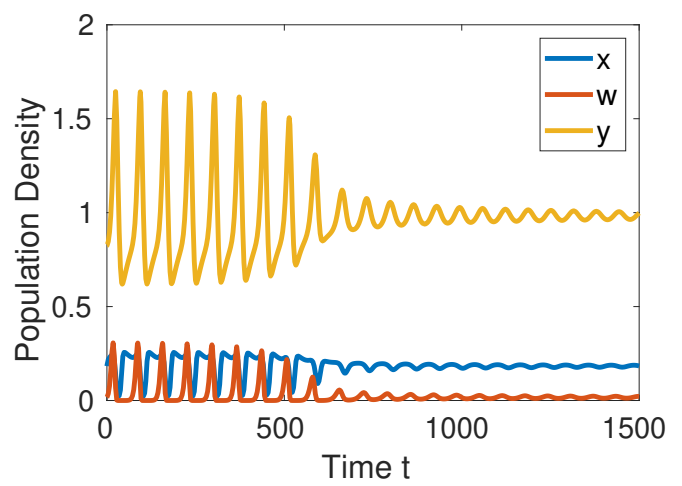

(a) Dynamics leading to a positive steady state.

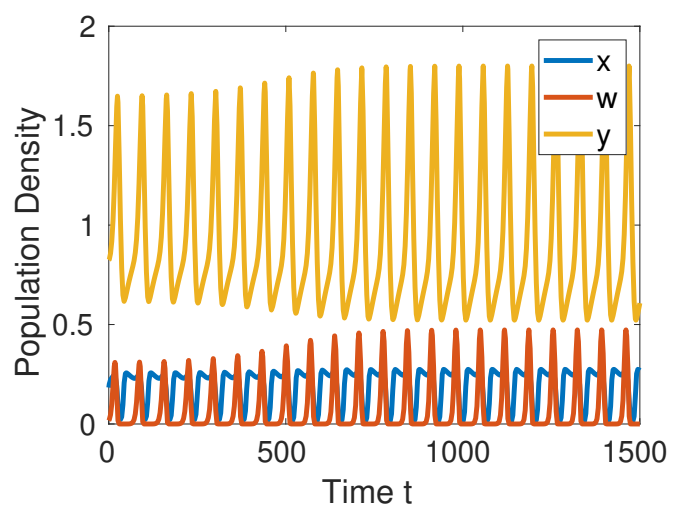

(b) Dynamics leading to a stable limit cycle.

Figure 4: Simulations of the full predator-two prey model (5). The parameter values $R=1, \Phi=2, D=0.1, K_{1}=0.36, K_{2}=1, P=5, Q=5$ are fixed. Both simulations are at the positive steady state $x^{*} \approx 0.1831, w^{*} \approx$ $0.0169, y^{*} \approx 0.9831$, with a small perturbation added to the initial predator population. In (a) we set $y(0)=0.825$ and in (b) we set $y(0)=0.824$.

example of a three-species limit cycle which occurs for the same parameter values at which a positive steady state is stable. The positive steady state is locally stable, and trajectories which initially are within the basin of attraction tend to this steady state. However, when the initial condition is sufficiently far away from this steady state, the solutions tend to limit cycles. This means that for some parameter values, population evolution under (5) involve a hysteresis, with the initial state of the populations influencing their long-time dynamics. We 


\begin{tabular}{|c|c|c|}
\hline Behavior & $\left(x^{*}, s^{*}, y^{*}\right)$ & Feasibility \\
\hline Extinction & $(0,0,0)$ & $I=0$ \\
\hline Subsidy Only & $\left(0, \frac{I}{\Gamma}, 0\right)$ & $I, \Gamma>0$ \\
\hline Prey Only & $(K, 0,0)$ & $I=0$ \\
\hline Subsidy-Free & $\left(\frac{D}{1-D P}, 0, \frac{K-P D K-D}{K(1-D P)^{2}}\right)$ & $I=0,1-D P>0, K>\frac{D}{1-D P}$ \\
\hline Prey-Free & $\left(0, \frac{D}{1-D Q}, \frac{I}{D \Phi}-\frac{\Gamma}{\Phi(1-D Q)}\right)$ & $1-D Q>0, \frac{I}{\Gamma}>\frac{D}{1-D Q}$ \\
\hline Predator-Free & $\left(K, \frac{I}{\Gamma}, 0\right)$ & $I, \Gamma>0$ \\
\hline Positive & $\left(x^{*}, s^{*}, y^{*}\right)$ & $x^{*}>0, s^{*}>0, y^{*}>0$ \\
\hline
\end{tabular}

Table 5: Steady states and feasibility conditions for the primary predator-prey-subsidy model (8).

remark that such multistability phenomena has been observed in other ecological systems (Aguirre et al., 2009; Broer et al., 2007; González-Yanez et al., 2007; Tyson and Lutscher, 2016; Vayenas and Pavlou, 1999).

Although it is not as biologically relevant as the $P, Q=O(1)$ case, the $P, Q=0$ limit for (5) gives

$$
\begin{gathered}
\frac{\mathrm{d} x}{\mathrm{~d} t}=x\left(1-\frac{x}{K_{1}}-y\right), \\
\frac{\mathrm{d} w}{\mathrm{~d} t}=w\left(R-\frac{R}{K_{2}} w-\Phi y\right), \\
\frac{\mathrm{d} y}{\mathrm{~d} t}=y(x+w-D),
\end{gathered}
$$

We summarize the dynamics of the system (6) in Table 4. Importantly, only steady states are stable in this model, with the variety of non-equilibrium dynamics emergent from (5) suppressed due to the Type I rather than Type II functional response. The asymmetric steady states found for the model (5) when the preference parameter $\Phi \neq 1$ are retained in this simpler model. Despite the drastic simplification $P, Q=0$, (6) preserves the variety of steady state dynamics seen under the more complicated model (5), although the former does not have the non-equilibrium dynamics of the latter.

\section{Non-Kolmogorov model for generalist predators utiliz- ing prey and allochthonous resource}

We now consider the case of a generalist predator exploiting a prey and an allochthonous resource. For example, while interactions between the Arctic fox and the lemming have been considered as a prototypical predator-prey system (Angerbjorn et al., 1999), it is known that the Arctic fox also consumes birds and bird eggs, and scavenges seal carrion discarded by polar bears on the sea ice (Roth, 2002, 2003). Similar interaction patterns have also been observed in systems where river otters predate on fish and plants (Ben-David et al., 1998), systems where wolves predate on both deer and salmon carcasses (Adams et al., 2010; Darimont et al., 2008), systems where puma exploit both mule deer and mule deer carcasses (Bauer et al., 2005), and also systems where Kodiak brown bears predate on Pacific salmon and berries (Barnes Jr, 1990; Berns and Hensel, 1972; Berns et al., 1980; Reimchen, 2000; Willson, 1993). Motivated by some of these ecological systems, Nevai and Van Gorder (2012) extended the Rosenzweig-MacArthur model (1) to a predator-prey-subsidy model of the form

$$
\begin{gathered}
\frac{\mathrm{d} x}{\mathrm{~d} t}=r x\left(1-\frac{x}{k}\right)-\frac{\theta x y}{x+s+h}, \\
\frac{\mathrm{d} s}{\mathrm{~d} t}=i-\gamma s-\frac{\psi s y}{x+s+h}, \\
\frac{\mathrm{d} y}{\mathrm{~d} t}=\frac{\epsilon \theta x y}{x+s+h}+\frac{\eta \psi s y}{x+s+h}-\delta y,
\end{gathered}
$$

where $s$ denotes the population of the subsidy, $i$ is the subsidy input rate, $\gamma$ is the subsidy decay rate, $\psi$ is the maximum rate at which the predator consumes the subsidy, $\eta$ is the conversion rate of the energy that the predator obtains from the subsidy, and the other parameters are the same as in the RosenzweigMacArthur model (1). Note that the input rate $i$ is due to an exogenous source, and hence this model is an example of a non-Kolmogorov generalist predator model. Dynamics from this form of the model were studied in Nevai and Van Gorder (2012).

To non-dimensionalize (7), we take $t=\bar{t} \hat{t}, x=\bar{x} \hat{x}, y=\bar{y} \hat{y}$, $s=\bar{s} \hat{s}$, where the scalings are $\bar{t}=\frac{1}{r}, \bar{y}=\frac{h r}{\theta}, \bar{x}=\frac{h r}{\epsilon \theta}, \bar{s}=\frac{h r}{\eta \psi}$, and also define the dimensionless groups $K=\frac{\epsilon \theta k}{h r}, I=\frac{i \eta \psi}{h r^{2}}$, $\Gamma=\frac{\gamma}{r}, \Phi=\frac{\psi}{\theta}, D=\frac{\delta}{r}, P=\frac{r}{\epsilon \theta}, Q=\frac{r}{\eta \psi}$. Here $K=O(1)$ is the effective carrying capacity, $I=O(1)$ is the effective input rate of the subsidy, $\Gamma \ll 1$ is the ratio of the renewal rate of the prey to the decay rate of the subsidy, $D \ll 1$ is the effective death rate, $P, Q=O(1)$ are the ratios of renewal rate to exploitation rate for the prey and subsidy, respectively, and $\Phi$ is again the preference parameter, with $\Phi<1$ if the prey is preferred over the subsidy, and $\Phi>1$ if the subsidy is preferred over the prey. Dropping hats, the dimensionless form of (7) reads

$$
\begin{gathered}
\frac{\mathrm{d} x}{\mathrm{~d} t}=x\left(1-\frac{x}{K}-\frac{y}{1+P x+Q s}\right), \\
\frac{\mathrm{d} s}{\mathrm{~d} t}=I-s\left(\Gamma+\frac{\Phi y}{1+P x+Q s}\right), \\
\frac{\mathrm{d} y}{\mathrm{~d} t}=y\left(\frac{x+s}{1+P x+Q s}-D\right) .
\end{gathered}
$$

One can show that the dynamics of (8) are bounded, and in Table 5 we list steady states and corresponding feasibility conditions. The stability criteria are more complicated, so as before we numerically determine the eigenvalues of the Jacobian 


\begin{tabular}{|c|c|c|c|}
\hline Behavior & $\left(x^{*}, s^{*}, y^{*}\right)$ & Feasibility & Stability \\
\hline Extinction & $(0,0,0)$ & $I=0$ & Never \\
\hline Subsidy Only & $\left(0, \frac{I}{\Gamma}, 0\right)$ & $I, \Gamma>0$ & Never \\
\hline Prey Only & $(K, 0,0)$ & $I=0$ & $D>K$ \\
\hline Subsidy-Free & $\left(D, 0,1-\frac{D}{K}\right)$ & $I=0, D<K$ & When Feasible \\
\hline Prey-Free & $\left(0, D, \frac{I-D \Gamma}{D \Phi}\right)$ & $I-D \Gamma>0$ & $I>D(\Gamma+\Phi)$ \\
\hline Predator-Free & $\left(K, \frac{I}{\Gamma}, 0\right)$ & $I, \Gamma>0$ & $D>K+\frac{I}{\Gamma}$ \\
\hline Positive & $\left(x^{*}, s^{*}, y^{*}\right)$ & $-\Gamma K<I-D \Gamma<D \Phi$ & When Feasible \\
\hline
\end{tabular}

Table 6: Steady states, feasibility and stability conditions for the simplified predator-prey-subsidy model (9). Note that the feasibility conditions for the positive steady state are the complement of the stability conditions for the other steady states. We show that the positive steady state is stable when feasible, hence there will be exactly one stable steady state for all parameter values.

matrix for each steady state, and use this to construct bifurcation diagrams. Since the subsidy-only steady state is always unstable, and the extinction, prey-only, and subsidy-free steady states are only stable on the $K$-axis (where $I=0$ ), only the predator-free, prey-free, and positive steady states will appear in our bifurcation diagrams.

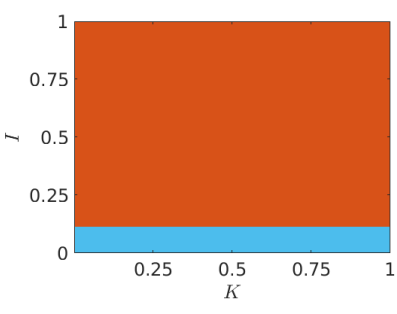

(a) $P=1, Q=1$

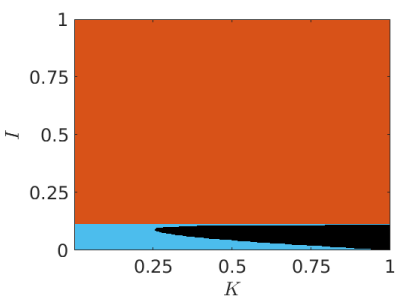

(c) $P=8, Q=1$

Predator-free SS
Prey-free SS

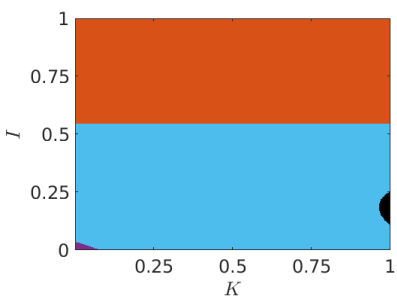

(b) $P=1, Q=8$

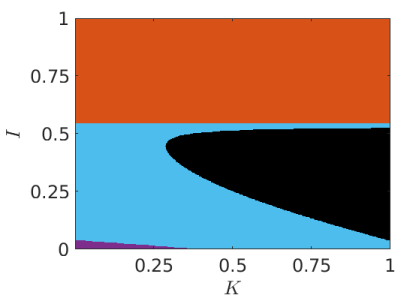

(d) $P=8, Q=8$
Positive SS

Non-equilibrium dynamics

Figure 5: Bifurcation diagrams over $K, I$ for the steady states and nonequilibrium dynamics of the full predator-prey-subsidy model (8) given various $P, Q$. We fix $D=0.1, \Gamma=0.1, \Phi=1$.

Figure 5 gives bifurcation diagrams for various values of the ratios of prey $(P)$ or subsidy $(Q)$ renewal to exploitation, given that the predator has the same preference for each. In this case, it is the rate parameter corresponding to the prey, $P$, which most strongly modifies the existence region of non-equilibrium dynamics. Given an increase in the ratio of prey renewal to exploitation, the stability region for the positive steady state decreases in area, while the regions for both the predator-free steady state and the non-equilibrium solutions increase in area. Meanwhile, an increase in the rate parameter corresponding to the subsidy results in a decrease in the stability region for the prey-free steady state, with an increase in the other two regions.

Unlike for the predator - two prey model (5), the preference parameter, $\Phi$, does not drastically change the qualitative structure of the bifurcation diagrams for the predator - prey - subsidy model (8), as we show in Figure 6. Rather, the structure of the bifurcation diagrams remains the same, with only quantitative shifts in the boundaries between regions apparent. This is due to the difference in functional response in the subsidy equation versus those of the predator and prey equations, highlighting one difference of including an exogenous source term modelled by a non-Kolmogorov framework.

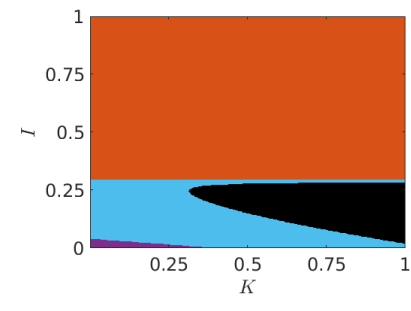

(a) $\Phi=0.5$

Predator-free SS

Prey-free SS

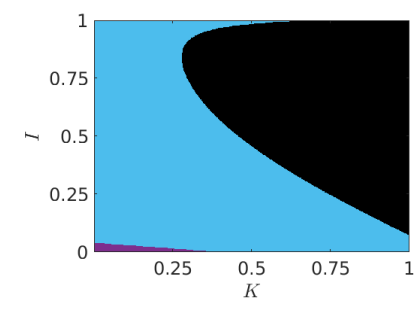

(b) $\Phi=2$
Positive SS

Non-equilibrium dynamics

Figure 6: Bifurcation diagrams over $K, I$ for the steady states and nonequilibrium dynamics of the full predator-prey-subsidy model (8) given various $P, Q$, and $\Phi$. We fix $D=0.1, \Gamma=0.1, P=Q=8$. Compare these with panel (d) of Figure 5 for $\Phi=1$, with all other parameters the same.

As we did in Sections 2-3, we take the $P, Q \rightarrow 0$ limit of (8), obtaining the Lotka-Volterra type model

$$
\begin{aligned}
\frac{\mathrm{d} x}{\mathrm{~d} t} & =x\left(1-\frac{x}{K}-y\right), \\
\frac{\mathrm{d} s}{\mathrm{~d} t} & =I-s(\Gamma+\Phi y), \\
\frac{\mathrm{d} y}{\mathrm{~d} t} & =y(x+s-D) .
\end{aligned}
$$

We summarize our analysis of this system in Table 6. The simplified predator-prey-subsidy model always admits a steady state solution, without the possibility of limit cycles. As the preference parameter increases, the region for prey-free steady states decreases in area. Therefore, as the preference of the predator is skewed toward the subsidy, this relieves pressure on 
the prey population, resulting in a wider range of parameters for coexistence. In contrast, if the input rate of the subsidy, $I$, is increased, then the prey may be worse off, as the predator will become more abundant, as we earlier observed in solutions to the model (8). Therefore, many of the qualitative findings for the steady states are the same despite the reduction of the Type II functional response to the Type I functional response, with the primary difference being the lack of non-equilibrium dynamics.

\section{Discussion}

We considered two classes of models for generalist predators which utilize more than one food source: predator - two prey models of Kolmogorov form, and predator - prey - subsidy models, with the subsidy exogenously fed into the system, breaking the symmetry of a Kolmogorov system. Both models were non-dimensionalized in compatible manners, which allowed for a straightforward comparison between the two models.

Understanding the region of parameter space permitting a positive steady state is important, as this state corresponds to the coexistence of predator and prey species which is often considered valuable from the perspective of maintaining species diversity. In the Kolmogorov predator-two prey model, increasing either of the ratio of prey renewal to exploitation $(P$ and $Q)$ resulted in an increase in the stability region for a positive steady state. In contrast, while this behavior was observed in the non-Kolmogorov predator-prey-subsidy model for the prey parameter $P$, the opposite behavior was noted when increasing the ratio of renewal to exploitation for the subsidy. This suggests that systems involving two prey species may yield dynamics which are more favorable for the survival of either prey species, compared with systems of only one prey species with the predator also exploiting a subsidy. Part of the reason is that the subsidy cannot go extinct, as it is input at a rate independent of the present values of subsidy in the system. This induces an asymmetry in the results, with large input rates of subsidy leading to larger predator populations, which in turn put more pressure on the prey population. In contrast, in the Kolmogorov model with two prey, excessive exploitation of either or both prey populations will result in fewer predators, allowing for a rebound in the respective prey populations. This is therefore one setting where the ecosystem may be more stable as a whole if the predator exploits two active prey, rather than an inert subsidy.

In the predator-two prey model, the value of the predator preference for each prey, $\Phi$, will significantly influence the dynamics. When the predator has a strong preference for one prey species over the other, this results in an asymmetry in both the emergent steady states and the non-equilibrium dynamics, and if the difference in preference is strong, then one of the two prey species may be pushed to extinction. Solutions in this extreme involve either steady states with only two positive populations, or limit cycles involving only two populations, with the third population dying off to zero. In contrast, there was no appreciable qualitative change in the dynamics observed under the predator - prey - subsidy model with a change in the respective preference parameter. In this sense, the non-Kolmogorov model maintains the general dynamics despite a shift in predator preferences, whereas a shift in predator preferences may result in drastic changes to the food web modelled by the predator - two prey Kolmogorov model. As such, inclusion of a subsidy may make for a more stable ecosystem in cases where the predator preferences are highly skewed. With that said, excessive input rates of subsidy, I, can lead to rapid growth of predator populations, which puts added stress on the prey and may lead to prey extinction (consistent with findings in Nevai and Van Gorder (2012)).

For certain restricted parameter values, we saw that initial conditions may determine the long-time dynamics in addition to the model parameters under the Kolmogorov model, with steady states and limit cycles being simultaneously locally stable for identical parameter values with different initial conditions. This situation is referred to as multistability, with two or more states being locally stable, and no one state being globally stable. Multistability has been observed in other nonlinear models of population dynamics (Aguirre et al., 2009; Broer et al., 2007; González-Yanez et al., 2007; Tyson and Lutscher, 2016; Vayenas and Pavlou, 1999) and hence this finding is not completely surprising. Such dynamics were not present in the non-Kolmogorov predator - prey - subsidy system, and hence the initial conditions did not play any role in long-time dynamics of that system provided all initial conditions were positive. This suggests that there is a degree of hysteresis inherent in the three-species Kolmogorov model which is not present in the non-Kolmogorov model due to symmetry breaking. While this dependence on initial conditions is somewhat troubling from a modelling point of view, it is not common for the parameter choices we investigated, being confined to small sets of parameters where stable steady states and stable limit cycles exist simultaneously. Still, this hysteresis is useful to be aware of as a possible source of error in long-time predictions of population dynamics under such Kolmogorov models.

We also obtained reduced or simplified forms of each of the full models, assuming that renewal rate of the prey or subsidy (as appropriate) was small relative to the exploitation rate of each resource by the predator. In this limit, the Type II functional response terms reduce to Type I functional response terms. While perhaps less relevant to most ecosystems, this provides a reduction of each model to a type of Lotka-Volterra system, which is simpler to work with and perhaps useful for pedagogical reasons. These simplified models exhibit all of the various steady states seen in the models with Type II response, yet they do not exhibit the non-equilibrium dynamics. This finding is in contrast to different Lotka-Volterra models considered in the literature for three or more species (Hofbauer, 1981; Zeeman, 1993), with the difference being that there is no direct interaction term between either of the two prey species in our models. As discussed in Fujii (1977), such competition between the two prey species is needed to drive limit cycles in predator-prey forms of three species Lotka-Volterra models, so our finding is not surprising.

The models we study can be made more realistic if we take 
additional environmental factors into consideration. One option is to have distinct handling times for each food source, or to make these handling times, or other parameters, time dependent. Indeed, motivated by the seasonal diet of polar bears (Thiemann et al., 2008) and seasonal changes in the intrinsic growth rate and the carrying capacity of lemmings (Turchin and Hanski, 1997), Levy et al. (2016) explored predator-preysubsidy dynamics where the constant input rate and decay rate of the subsidy, the intrinsic growth rate of the prey and the predation rate of the predator were time-dependent. Even for simpler predator-prey models, seasonal changes can cause chaotic behaviors (Fuentes Sommer, 2016; Rinaldi et al., 1993), and such time dependent parameters would likely modify some of the dynamics we observe. Spatially extended non-Kolmogorov models have been studied in a variety of contexts, motivated by the Arctic fox - lemming - seal carrion (Bassett et al., 2017; Eide et al., 2018; Jansen and Van Gorder, 2018; Shen and Van Gorder, 2017), as well as Kodiak Brown Bear - Pacific Salmon predator - prey system in which the bears also consume berries as an allochthonous resource (Fussell et al., 2019). Further systematic classification of model dynamics across different spatial habitats could provide valuable insight into the spatiotemporal structure of ecosystems involving generalist predation. Indeed, for the Kolmogorov model, asymmetric states may be replaced with states where one prey survives in one region of the habitat, with the other prey surviving in a distinct region.

\section{References}

Abrams, P., Matsuda, H., 1993. Effects of adaptive predatory and anti-predator behaviour in a two-prey-one-predator system. Evolutionary Ecology 7 (3), 312-326.

Abrams, P. A., 2006. The effects of switching behavior on the evolutionary diversification of generalist consumers. The American Naturalist 168 (5), 645-659.

Adams, L. G., Farley, S. D., Stricker, C. A., Demma, D. J., Roffler, G. H., Miller, D. C., Rye, R. O., 2010. Are inland wolf-ungulate systems influenced by marine subsidies of pacific salmon? Ecological Applications 20 (1), 251-262.

Aguirre, P., González-Olivares, E., Sáez, E., 2009. Three limit cycles in a leslie-gower predator-prey model with additive allee effect. SIAM Journal on Applied Mathematics 69 (5), 1244-1262.

Angerbjorn, A., Tannerfeldt, M., Erlinge, S., 1999. Predator-prey relationships: arctic foxes and lemmings. Journal of Animal Ecology 68 (1), 34-49.

Barnes Jr, V. G., 1990. The influence of salmon availability on movements and range of brown bears on southwest Kodiak Island. Bears: Their Biology and Management, 305-313.

Bassett, A., Krause, A. L., Van Gorder, R. A., 2017. Continuous dispersal in a model of predator-prey-subsidy population dynamics. Ecological Modelling 354, 115-122.

Bauer, J. W., Logan, K. A., Sweanor, L. L., Boyce, W. M., 2005. Scavenging behavior in puma. The Southwestern Naturalist 50 (4), 466-471.

Ben-David, M., Bowyer, R., Duffy, L., Roby, D., Schell, D., 1998. Social behavior and ecosystem processes: river otter latrines and nutrient dynamics of terrestrial vegetation. Ecology 79 (7), 2567-2571.

Berns, V. D., Atwell, G. C., Boone, D. L., 1980. Brown bear movements and habitat use at Karluk Lake, Kodiak Island. Bears: Their Biology and Management, 293-296.

Berns, V. D., Hensel, R. J., 1972. Radio tracking brown bears on kodiak island. Bears: Their Biology and Management, 19-25.

Broer, H., Saleh, K., Naudot, V., Roussarie, R., 2007. Dynamics of a predatorprey model with non-monotonic response function. Discrete \& Continuous Dynamical Systems-A 18 (2/3), 221-251.
Clare, E. L., Fraser, E. E., Braid, H. E., Fenton, M. B., Hebert, P. D., 2009. Species on the menu of a generalist predator, the eastern red bat (lasiurus borealis): using a molecular approach to detect arthropod prey. Molecular ecology 18 (11), 2532-2542.

Darimont, C. T., Paquet, P. C., Reimchen, T. E., 2008. Spawning salmon disrupt trophic coupling between wolves and ungulate prey in coastal british columbia. BMC Ecology 8 (1), 14.

Dhooge, A., Govaerts, W., Kuznetsov, Y. A., 2003. Matcont: a matlab package for numerical bifurcation analysis of odes. ACM Transactions on Mathematical Software (TOMS) 29 (2), 141-164.

Díaz-Ruiz, F., Delibes-Mateos, M., García-Moreno, J. L., María López-Martín, J., Ferreira, C., Ferreras, P., 2013. Biogeographical patterns in the diet of an opportunistic predator: the red fox $\mathrm{v}$ ulpes vulpes in the $\mathrm{i}$ berian $\mathrm{p}$ eninsula. Mammal Review 43 (1), 59-70.

Doedel, E. J., 1981. Auto: A program for the automatic bifurcation analysis of autonomous systems. Congr. Numer 30 (265-284), 25-93.

Eide, R. M., Krause, A. L., Fadai, N. T., Van Gorder, R. A., 2018. Predatorprey-subsidy population dynamics on stepping-stone domains with dispersal delays. Journal of Theoretical Biology 451, 19-34.

El-Gohary, A., Al-Ruzaiza, A., 2007. Chaos and adaptive control in two prey, one predator system with nonlinear feedback. Chaos, Solitons \& Fractals 34 (2), 443-453.

Erbach, A., Lutscher, F., Seo, G., 2013. Bistability and limit cycles in generalist predator-prey dynamics. Ecological complexity 14, 48-55.

Fisher, J. T., Wilkinson, L., 2005. The response of mammals to forest fire and timber harvest in the north american boreal forest. Mammal Review 35 (1), 51-81.

Fuentes Sommer, P. E., 2016. Analysis of the rosenzweig-macarthur model with bifurcation structures and stochastic processes. Ph.D. thesis.

Fujii, K., 1977. Complexity-stability relationship of two-prey-one-predator species system model: Local and global stability. Journal of Theoretical Biology 69 (4), 613-623.

Fussell, E. F., Krause, A. L., Van Gorder, R. A., 2019. Hybrid approach to modeling spatial dynamics of systems with generalist predators. Journal of theoretical biology 462, 26-47.

Gakkhar, S., et al., 2003. Existence of chaos in two-prey, one-predator system. Chaos, Solitons \& Fractals 17 (4), 639-649.

Genkai-Kato, M., Yamamura, N., 2000. Profitability of prey determines the response of population abundances to enrichment. Proceedings of the Royal Society of London B: Biological Sciences 267 (1460), 2397-2401.

Gilpin, M. E., 1979. Spiral chaos in a predator-prey model. The American Naturalist 113 (2), 306-308.

Giroux, M.-A., Berteaux, D., Lecomte, N., Gauthier, G., Szor, G., Bêty, J., 2012. Benefiting from a migratory prey: spatio-temporal patterns in allochthonous subsidization of an arctic predator. Journal of Animal Ecology 81 (3), 533-542.

González-Yanez, B., González-Olivares, E., MENA-LORCA, J., 2007. Multistability on a leslie-gower type predator-prey model with nonmonotonic functional response. In: BIOMAT 2006. World Scientific, pp. 359-384.

Grasman, J., Van Den Bosch, F., Van Herwaarden, O., 2001. Mathematical conservation ecology: A one-predator-two-prey system as case study. Bulletin of Mathematical Biology 63 (2), 259-269.

Halaj, J., Wise, D. H., 2002. Impact of a detrital subsidy on trophic cascades in a terrestrial grazing food web. Ecology 83 (11), 3141-3151.

Hamilton, W., 1935. Notes on food of red foxes in new york and new england. Journal of Mammalogy 16 (1), 16-21.

Hastings, A., Powell, T., 1991. Chaos in a three-species food chain. Ecology 72 (3), 896-903.

Henden, J.-A., Ims, R. A., Yoccoz, N. G., Hellström, P., Angerbjörn, A., 2010. Strength of asymmetric competition between predators in food webs ruled by fluctuating prey: the case of foxes in tundra. Oikos 119 (1), 27-34.

Hins, C., Ouellet, J.-P., Dussault, C., St-Laurent, M.-H., 2009. Habitat selection by forest-dwelling caribou in managed boreal forest of eastern canada: evidence of a landscape configuration effect. Forest Ecology and Management 257 (2), 636-643.

Hobbs, N. T., Swift, D. M., 1985. Estimates of habitat carrying capacity incorporating explicit nutritional constraints. The Journal of Wildlife Management, 814-822.

Hofbauer, J., 1981. On the occurrence of limit cycles in the volterra-lotka equation. Nonlinear Analysis: Theory, Methods \& Applications 5 (9), 1003 1007. 
Hofbauer, J., Sigmund, K., 1998. Evolutionary games and population dynamics. Cambridge University Press.

Holling, C. S., 1959. The components of predation as revealed by a study of small-mammal predation of the european pine sawfly. The Canadian Entomologist 91 (5), 293-320.

Holling, C. S., 1966. The functional response of invertebrate predators to prey density. The Memoirs of the Entomological Society of Canada 98 (S48), $5-86$.

Houle, M., Fortin, D., Dussault, C., Courtois, R., Ouellet, J.-P., 2010. Cumulative effects of forestry on habitat use by gray wolf (canis lupus) in the boreal forest. Landscape ecology 25 (3), 419-433.

Hsu, S.-B., 1978. On global stability of a predator-prey system. Mathematical Biosciences 39 (1-2), 1-10.

Hutson, V., Vickers, G., 1983. A criterion for permanent coexistence of species, with an application to a two-prey one-predator system. Mathematical Biosciences 63 (2), 253-269.

Jansen, J. E., Van Gorder, R. A., 2018. Dynamics from a predator-prey-quarryresource-scavenger model. Theoretical Ecology 11 (1), 19-38.

Kar, T., Ghosh, B., 2012. Sustainability and optimal control of an exploited prey predator system through provision of alternative food to predator. Biosystems 109 (2), 220-232.

Klebanoff, A., Hastings, A., 1994. Chaos in one-predator, two-prey models: cgeneral results from bifurcation theory. Mathematical Biosciences 122 (2), 221-233.

Kolmogorov, A., 1936. Sulla teoria di volterra della lotta per lesistenza. Gi. Inst. Ital. Attuari 7, 74-80.

Kot, M., 2001. Elements of mathematical ecology. Cambridge University Press. Kumar, S., Srivastava, S., Chingakham, P., 2002. Hopf bifurcation and stability analysis in a harvested one-predator-two-prey model. Applied Mathematics and Computation 129 (1), 107-118.

Leeuwen, E. v., Jansen, V., Bright, P., 2007. How population dynamics shape the functional response in a one-predator-two-prey system. Ecology 88 (6), 1571-1581.

Legagneux, P., Gauthier, G., Berteaux, D., Bêty, J., Cadieux, M.-C., Bilodeau, F., Bolduc, E., McKinnon, L., Tarroux, A., Therrien, J.-F., et al., 2012. Disentangling trophic relationships in a high arctic tundra ecosystem through food web modeling. Ecology 93 (7), 1707-1716.

Levy, D., Harrington, H. A., Van Gorder, R. A., 2016. Role of seasonality on predator-prey-subsidy population dynamics. Journal of Theoretical Biology 396, 163-181.

Liu, M., Wang, K., 2013. Dynamics of a two-prey one-predator system in random environments. Journal of Nonlinear Science 23 (5), 751-775.

Marczak, L. B., Thompson, R. M., Richardson, J. S., 2007. Meta-analysis: trophic level, habitat, and productivity shape the food web effects of resource subsidies. Ecology 88 (1), 140-148.

Mariani, P., Andersen, K. H., Visser, A. W., Barton, A. D., Kiørboe, T., 2013. Control of plankton seasonal succession by adaptive grazing. Limnology and Oceanography 58 (1), 173-184.

Maron, J. L., Pearson, D. E., Fletcher Jr, R. J., 2010. Counterintuitive effects of large-scale predator removal on a midlatitude rodent community. Ecology 91 (12), 3719-3728.

Messelink, G. J., van Maanen, R., van Steenpaal, S. E., Janssen, A., 2008. Biological control of thrips and whiteflies by a shared predator: two pests are better than one. Biological Control 44 (3), 372-379.

Morozov, A., Petrovskii, S., 2013. Feeding on multiple sources: towards a universal parameterization of the functional response of a generalist predator allowing for switching. PloS one 8 (9), e74586.

Mougi, A., 2010. Coevolution in a one predator-two prey system. PloS one 5 (11), e13887.

Mougi, A., Nishimura, K., 2008. The paradox of enrichment in an adaptive world. Proceedings of the Royal Society of London B: Biological Sciences 275 (1651), 2563-2568.

Nevai, A. L., Van Gorder, R. A., 2012. Effect of resource subsidies on predatorprey population dynamics: a mathematical model. Journal of Biological Dynamics $6(2), 891-922$.

Nomdedeu, M. M., Willen, C., Schieffer, A., Arndt, H., 2012. Temperaturedependent ranges of coexistence in a model of a two-prey-one-predator microbial food web. Marine biology 159 (11), 2423-2430.

Nomikou, M., Sabelis, M. W., Janssen, A., 2010. Pollen subsidies promote whitefly control through the numerical response of predatory mites. Biocontrol 55 (2), 253-260.
Parshad, R. D., Bhowmick, S., Quansah, E., Basheer, A., Upadhyay, R. K., 2016. Predator interference effects on biological control: The "paradox" of the generalist predator revisited. Communications in Nonlinear Science and Numerical Simulation 39, 169-184.

Reimchen, T., 2000. Some ecological and evolutionary aspects of bear-salmon interactions in coastal british columbia. Canadian Journal of Zoology 78 (3), 448-457.

Rinaldi, S., Muratori, S., Kuznetsov, Y., 1993. Multiple attractors, catastrophes and chaos in seasonally perturbed predator-prey communities. Bulletin of Mathematical Biology 55 (1), 15-35.

Rose, M. D., Polis, G. A., 1998. The distribution and abundance of coyotes: the effects of allochthonous food subsidies from the sea. Ecology 79 (3), 998-1007.

Rosenzweig, M. L., 1971. Paradox of enrichment: destabilization of exploitation ecosystems in ecological time. Science 171 (3969), 385-387.

Rosenzweig, M. L., MacArthur, R. H., 1963. Graphical representation and stability conditions of predator-prey interactions. The American Naturalist 97 (895), 209-223.

Roth, J. D., 2002. Temporal variability in arctic fox diet as reflected in stablecarbon isotopes; the importance of sea ice. Oecologia 133 (1), 70-77.

Roth, J. D., 2003. Variability in marine resources affects arctic fox population dynamics. Journal of Animal Ecology 72 (4), 668-676.

Rutz, C., Bijlsma, R. G., 2006. Food-limitation in a generalist predator. Proceedings of the Royal Society of London B: Biological Sciences 273 (1597), 2069-2076.

Sanders, D., Schaefer, M., Platner, C., Griffiths, G. J., 2011. Intraguild interactions among generalist predator functional groups drive impact on herbivore and decomposer prey. Oikos 120 (3), 418-426.

Schaffer, W. M., Kot, M., 1986. Chaos in ecological systems: the coals that newcastle forgot. Trends in Ecology \& Evolution 1 (3), 58-63.

Seydel, R., 2009. Practical bifurcation and stability analysis. Vol. 5. Springer Science \& Business Media.

Shen, L., Van Gorder, R. A., 2017. Predator-prey-subsidy population dynamics on stepping-stone domains. Journal of Theoretical Biology 420, 241-258.

Sigmund, K., 2007. Kolmogorov and population dynamics. In: Kolmogorov's heritage in mathematics. Springer, pp. 177-186.

Smout, S., Asseburg, C., Matthiopoulos, J., Fernández, C., Redpath, S., Thirgood, S., Harwood, J., 2010. The functional response of a generalist predator. PLoS One 5 (5), e10761.

Snyder, W. E., Wise, D. H., 1999. Predator interference and the establishment of generalist predator populations for biocontrol. Biological Control 15 (3), 283-292.

Sunde, P., Thorup, K., Jacobsen, L. B., Rahbek, C., 2014. Weather conditions drive dynamic habitat selection in a generalist predator. PloS one 9 (2), e88221.

Sweitzer, R. A., Jenkins, S. H., Berger, J., 1997. Near-extinction of porcupines by mountain lions and consequences of ecosystem change in the great basin desert. Conservation Biology 11 (6), 1407-1417.

Symondson, W., Sunderland, K., Greenstone, M., 2002. Can generalist predators be effective biocontrol agents? Annual review of entomology 47 (1), 561-594.

Takeuchi, Y., Adachi, N., 1983. Existence and bifurcation of stable equilibrium in two-prey, one-predator communities. Bulletin of Mathematical Biology 45 (6), 877-900.

Terraube, J., Arroyo, B., 2011. Factors influencing diet variation in a generalist predator across its range distribution. Biodiversity and Conservation 20 (10), 2111-2131.

Thiemann, G. W., Iverson, S. J., Stirling, I., 2008. Polar bear diets and arctic marine food webs: insights from fatty acid analysis. Ecological Monographs 78 (4), 591-613.

Toyokawa, W., 2017. Scrounging by foragers can resolve the paradox of enrichment. Royal Society Open Science 4 (3), 160830.

Turchin, P., 2003. Complex population dynamics: a theoretical/empirical synthesis. Vol. 35. Princeton University Press.

Turchin, P., Hanski, I., 1997. An empirically based model for latitudinal gradient in vole population dynamics. The American Naturalist 149 (5), 842-874.

Tyson, R., Lutscher, F., 2016. Seasonally varying predation behavior and climate shifts are predicted to affect predator-prey cycles. The American Naturalist 188 (5), 539-553.

van Maanen, R., Vila, E., Sabelis, M. W., Janssen, A., 2010. Biological control of broad mites (polyphagotarsonemus latus) with the generalist predator 
amblyseius swirskii. Experimental and Applied Acarology 52 (1), 29-34.

Vance, R. R., 1978. Predation and resource partitioning in one predator-two prey model communities. The American Naturalist 112 (987), 797-813.

Vayenas, D., Pavlou, S., 1999. Chaotic dynamics of a food web in a chemostat. Mathematical Biosciences 162 (1-2), 69-84.

Weber, D. C., Lundgren, J. G., 2011. Effect of prior diet on consumption and digestion of prey and non-prey food by adults of the generalist predator coleomegilla maculata. Entomologia Experimentalis et Applicata 140 (2), 146-152.

Welch, K., Pfannenstiel, R., Harwood, J., 2012. The role of generalist predators in terrestrial food webs: lessons for agricultural pest management. Biodiversity and insect pests: key issues for sustainable management, 41-56.

Willson, J. D., Hopkins, W. A., 2011. Prey morphology constrains the feeding ecology of an aquatic generalist predator. Ecology 92 (3), 744-754.

Willson, M. F., 1993. Mammals as seed-dispersal mutualists in north america. Oikos, 159-176.

Wollkind, D. J., Collings, J. B., Logan, J. A., 1988. Temperature-mediated stability of the interaction between spider mites and predatory mites in orchards. Experimental \& Applied Acarology 5 (3-4), 265-292.

Yamauchi, A., Yamamura, N., 2005. Effects of defense evolution and diet choice on population dynamics in a one-predator-two-prey system. Ecology 86 (9), 2513-2524.

Zeeman, M. L., 1993. Hopf bifurcations in competitive three-dimensional lotka-volterra systems. Dynamics and Stability of Systems 8 (3), 189-216.

Zhang, X., Li, W., Liu, M., Wang, K., 2015. Dynamics of a stochastic holling ii one-predator two-prey system with jumps. Physica A: Statistical Mechanics and its Applications 421, 571-582. 